Ermittlung der Umweltschutzkosten bei VW

\section{Die Zahlen kennen}

Seit 1990 werden Betriebskosten für den Umweltschutz in der Volkswagen AG freiwillig erfasst; seit 1997 besteht dazu eine gesetzliche Verpflichtung. Die Erfassung und Zusammenstellung der Umweltschutzkosten ist bei einem großen Unternehmen mit erheblichem Aufwand verbunden. Gründe sind der Datenumfang und die Komplexität der betrieblichen Strukturen. Im Folgenden wird dargestellt, wie VW an den sechs inländischen Standorten mit diesen Problemen in der Praxis umgeht.

A

Von Ines Reischmann und Gerd-Peter Benthe bezeichnet man alle Kosten, die durch den Betrieb von Anlagen oder durch Maßnahmen entstehen, die dem Umweltschutz dienen. Seit 1997 müssen diese Angaben von großen Unternehmen gemäß §15 Umweltstatistikgesetz (UStatG) an das Statistische Bundesamt übermittelt werden. Darüber hinaus veröffentlicht die Volkswagen AG diese Umweltschutzkosten im Rahmen der Umweltberichterstattung und im Geschäftsbericht. Auch bei Versicherungen und Banken haben diese Daten zunehmende Bedeutung, Stichworte sind hier Haftungsrisiken und Ökofonds.

\section{Vom Meldesystem zu Kennzahlen}

Bei Volkswagen werden die Kosten nach dem Entstehungsprinzip erfasst, sodass eine verursachergerechte Zuordnung der Umweltschutzkosten nicht möglich ist. So werden zum Beispiel die Abfallentsorgungskosten eines Werkes nur einer Kostenstelle zugeordnet, aber nicht den verschiedenen Verursachern weiterbelastet. Außerdem gibt es für Anlagen, die dem Umweltschutz dienen, in der Regel keine eigenen Kostenstellen. Aus diesem Grund muss für ca. 2800 Kostenstellen in den deutschen Standorten der WW AG der Kostenanteil für den Umweltschutz separat ermittelt werden. Diese Aufgabe obliegt der zentralen Umweltschutzabteilung von Volkswagen.

Bis 1998 wurden die Umweltschutzbetriebskosten mit Hilfe eines Meldesystems ermittelt. Zu diesem Zweck wurde für jeden der nachstehenden Umweltschutzbereiche eine Nummer zur statistischen Erfassung eingerichtet:

1. Abfallwirtschaft,

2. Gewässerschutz,

3. Lärmbekämpfung,

4. Luftreinhaltung,
5. Naturschutz- und Landschaftspflege,

6 . Bodensanierung.

Alle Kostenbelege, die umweltschutzbedingte Vorgänge dokumentierten, mussten durch die Mitarbeiter mit der entsprechenden oben genannten Nummer gekennzeichnet werden. Nach denselben Kriterien wurden die Personalstunden, die im Rahmen des Umweltschutzes geleistet wurden, von jeder Kostenstelle quartalsweise gemeldet. In Verbindung mit verschiedenen Konten konnte eine Zuordnung zu Kostenarten, wie zum Beispiel Abschreibungen, Gebühren und Abgaben, vorgenommen werden. Diese dezentrale Methode verursachte jedoch einen sehr hohen Erfassungsaufwand. Aus diesem Grund wurde im Jahr 1994 versuchsweise in der mechanischen Fertigung des Werkes Wolfsburg die Möglichkeit geprüft, ob sich die Umweltschutzbetriebskosten bzw. Teile davon proportional zu den Kostenstellenkosten verhalten und inwiefern es möglich ist, diesen Kostenanteil in Form einer Kennzahl zu bestimmen. Die erste Voruntersuchung lieferte ein positives $\mathrm{Er}$ gebnis. Aufgrund der gewonnenen Erkenntnisse wurde von 1997 bis 1999 in den inländischen Standorten ein Kennzahlensystem zur Ermittlung der Umweltschutzkosten aufgebaut.

Abb. 1: Welche Kosten werden erfasst?

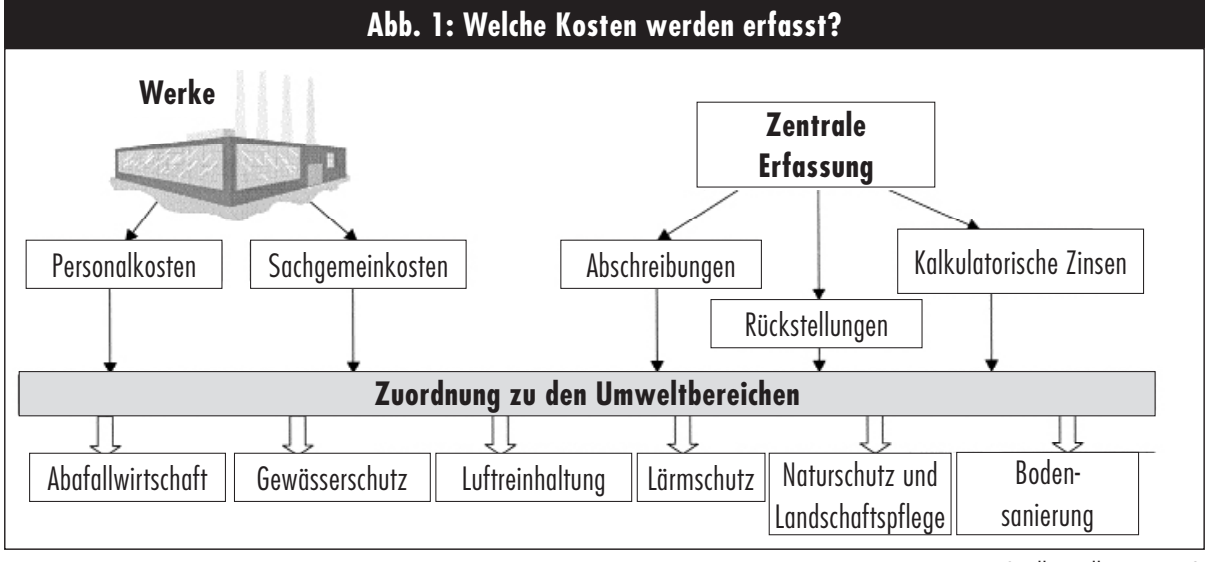

Quelle: Volkswagen AG 


\begin{tabular}{|l|c|c|}
\hline \multicolumn{2}{|c|}{ Abbildung 2: Vorteile des Kennzahlensystems } \\
\hline & altes Meldesystem & Kennzahlensystem \\
\hline Erfassungsmethode & dezentral & zentral \\
Beteiligte Mitarbeiter & sehr viele & wenige \\
Abgrenzung umweltrelevanter & uneinheitlich & einhtlich \\
Anlagen/MaßBnahmen & gering & hoch \\
Genauigkeit des Zahlenmaterials & hoch & gering \\
\hline $\begin{array}{l}\text { Erhebungsaufwand } \\
\text { nach Einführung }\end{array}$ & gering & hoch \\
\hline Flexibilität bei Änderungen & & \\
\hline
\end{tabular}

weisung vereinheitlichte die Ermittlung und vermied weitestgehend Abgrenzungsprobleme. Somit konnten die Kostenstellenmitarbeiter häufig die Kosten selbstständig erfassen. Bei komplexen oder schwierigen Untersuchungen erfolgte die Ermittlung der Umweltkosten in Zusammenarbeit mit den Mitarbeitern vor Ort und der zentralen Umweltschutzabteilung. Zu diesem Zweck waren Besichtigungen und Befragungen einzelner Mitarbeiter in den Fertigungsbereichen notwendig.

4. Ergebnisse. Anhand der ermittelten Umweltschutzbetriebskosten wurde der prozentuale Anteil an den Gesamtbetriebskosten als Kennzahl ermittelt. Die hierfür notwendigen Budgetinformationen wurden aus Systemen des Finanzwesens bereitgestellt. Die Kostenstellenbezeichnungen, Kennzahlen, Umweltschutzbetriebskosten, Gesamtbetriebskosten sowie die Aufteilung auf die einzelnen Umweltschutzbereiche wurden in einem Matrixsystem verarbeitet. Dieses Matrixsystem orientiert sich an den Kriterien des Erhebungsbogens des Statistischen Bundesamtes.

5. Flexible Anpassung der Kennzahlen. Die Pflege des Systems erfolgt durch die zentrale Umweltschutzabteilung der VW AG. Hierbei werden jährlich die Kennzahlen bei Struktur- oder Prozessveränderungen angepasst. Diese Informationen werden bei den örtlichen Umweltschutzabteilungen erfragt.

\section{Einen Überblick über alle bisher erschienenen Hefte des Informationsdienstes "Ökologisches Wirtschaften" finden Sie auf unseren Internetseiten unter der Adresse:}

\section{http://www.oekom.de}

\section{Erhebliche Vorteile}

Nach der aufwändigen Einführungsphase ist der administrative Aufwand erheblich geringer geworden als bei dem alten Meldesystem. Ebenso hat sich durch die einheitliche Erfassung und Abgrenzung die Zahlenqualität und -quantität verbessert. Zudem ist die Anpassungsfähigkeit dieses Erfassungssystems bei Veränderungen in den betrieblichen Bereichen sehr hoch (vgl. Abb. 2).

Untersuchungen haben ergeben, dass die Vergleichbarkeit und Übertragbarkeit der Kennzahlen in ähnlich strukturierten Bereichen mit vergleichbarer Anlagen- und Prozesstechnik gegeben sind. Beispielsweise können bei unterschiedlicher Aufgabenverteilung die Kennzahlen verschiedener mechanischer Fertigungen voneinander abweichen - je nachdem, ob die Instandhaltung zentral oder dezentral strukturiert ist. Als Steuerungsgröße sind die Kennzahlen allerdings nur wenig geeignet, $\mathrm{da}$ die Kosten bei Volkswagen nach dem Entstehungsprinzip erfasst werden.

Die zunehmende Berichtspflicht, die unter anderem aus gesetzlichen Anforderungen auf europäischer Ebene zu erwarten ist, macht die Ausweitung des Kennzahlensystems auf andere Standorte erforderlich. Derzeit führen die europäischen Standorte der Marke Volkswagen das neue System ein.

\section{Die AutorInnen}

Ines Reischmann und Gerd-Peter Benthe sind Mitarbeiter der Volkswagen AG.

Kontakt: Volkswagen AG, Umweltplanung Produktion/Standorte, Brieffach 1897, 38436 Wolfsburg, Tel. 05361/ 9-35455/ -74621, Fax -70687, E-Mail: ines.reischmann@volkswagen.de, gerd-peter.benthe@volkswagen.de

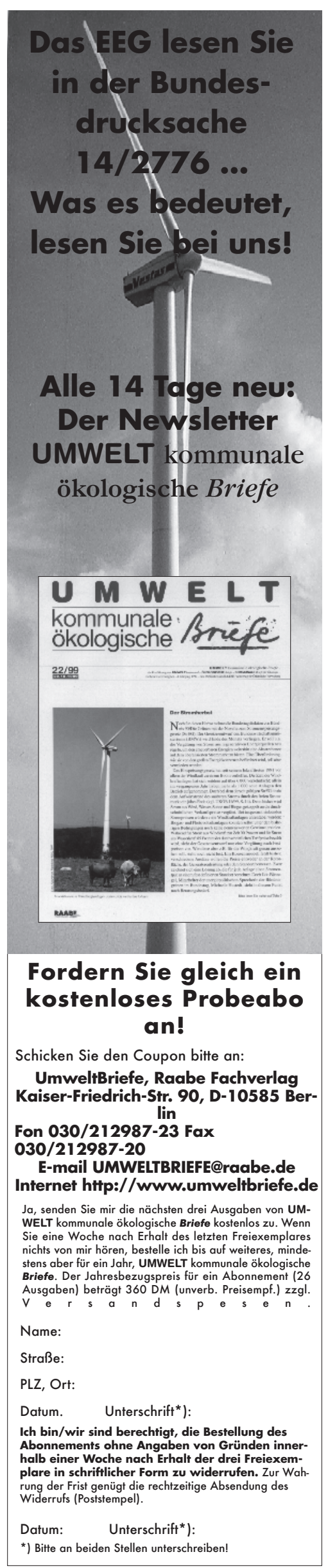


(c) 20I0 Authors; licensee IÖW and oekom verlag. This is an article distributed under the terms of the Creative Commons Attribution Non-Commercial No Derivates License (http://creativecommons.org/licenses/by-nc-nd/3.o/), which permits unrestricted use, distribution, and reproduction in any medium, provided the original work is properly cited. 